\title{
Ovarian Hormones Regulate the Production of Adipocytes From Bone Marrow-Derived Cells
}

\begin{abstract}
Kathleen M. Gavin ${ }^{1,2,3 *}$, Timothy M. Sullivan ${ }^{3,4,5}$, Wendy M. Kohrt ${ }^{1,3}$, Susan M. Majka ${ }^{6}$ and Dwight J. Klemm $m^{2,3,4,5}$

'Division of Geriatric Medicine, School of Medicine, University of Colorado Anschutz Medical Campus, Aurora, CO, United States, ${ }^{2}$ Charles C. Gates Center for Regenerative Medicine and Stem Cell Biology, School of Medicine, University of Colorado Anschutz Medical Campus, Aurora, CO, United States, ${ }^{3}$ Geriatric Research, Education and Clinical Center, VA Eastern Colorado Heath Care System, Denver, CO, United States, ${ }^{4}$ Division of Pulmonary Sciences and Critical Care Medicine, School of Medicine, University of Colorado Anschutz Medical Campus, Aurora, CO, United States, ${ }^{5}$ Cardiovascular Pulmonary Research Laboratory, School of Medicine, University of Colorado Anschutz Medical Campus, Aurora, CO, United States, ${ }^{6}$ Division of Allergy, Pulmonary, and Critical Care Medicine, Department of Medicine, Vanderbilt University School of Medicine, Nashville, TN, United States
\end{abstract}

OPEN ACCESS

Edited by:

Jennifer L. Miles-Chan, University of Auckland,

New Zealand

Reviewed by:

Kathleen Grace Mountjoy, University of Auckland,

New Zealand

Neil Youngson,

University of New South

Wales, Australia

${ }^{*}$ Correspondence:

Kathleen M. Gavin

kathleen.gavin@ucdenver.edu

Specialty section: This article was submitted to Obesity,

a section of the journal Frontiers in Endocrinology

Received: 14 March 2018 Accepted: 11 May 2018

Published: 28 May 2018

Citation:

Gavin KM, Sullivan TM, Kohrt WM, Majka SM and Klemm DJ (2018) Ovarian Hormones Regulate the Production of Adipocytes From Bone Marrow-Derived Cells.

Front. Endocrinol. 9:276. doi: 10.3389/fendo.2018.00276
Sex differences in body fat distribution and menopause-associated shifts in regional adiposity suggest that sex hormones play an important role in regulating the differentiation and distribution of adipocytes, but the underlying mechanisms have not been fully explained. The aim of this study was to determine whether ovarian hormone status influences the production and distribution of adipocytes in adipose tissue arising from bone marrow-derived cells. Nine- to ten-week-old ovariectomized (OVX), surgery naïve (WT), and estrogen receptor alpha knockout ( $\alpha E R K O)$ mice underwent bone marrow transplantation from luciferase or green fluorescent protein expressing donors. A subset of OVX animals had estradiol $\left(E_{2}\right)$ added back. Eight-weeks posttransplant, whole body and gonadal fat BM-derived adipocyte production was highest in OVX and $\alpha E R K O$ mice, which was attenuated in OVX mice by $E_{2}$ add-back. All groups demonstrated the highest bone marrow derived adipocyte (BMDA) production in the gonadal adipose depot, a visceral fat depot in mice. Taken together, the loss of ovarian hormones increases the production of BMDAs. If translatable across species, production of BMDA may be a mechanism by which visceral adiposity increases in estrogen-deficient postmenopausal women.

Keywords: adipocyte, estrogen receptor, bone marrow-derived cells, bone marrow transplant, ovarian hormones, myeloid cells

\section{INTRODUCTION}

Obesity is an ever-growing epidemic in the United States and around the world $(1,2)$. However, it is now clear that the disease risk conferred with obesity does not simply depend on overall adiposity, but on body fat distribution $(3,4)$. In fact, the cardiometabolic disease protection observed in women before the menopausal transition can be attributed, at least in part, to preferential accumulation of fat in peripheral adipose depots, primarily the hips and thighs (5). Notably, even without weight gain, peri- and postmenopausal women experience a shift in body fat distribution toward both subcutaneous and visceral abdominal adipose tissue depots $(6,7)$. Thus, sex hormones, and estrogen status, in particular, are hypothesized to play a mechanistic role in determining body fat 
distribution, although the specifics of this regulatory mechanism have yet to be completely described.

Depot-specific differences in gene expression and metabolic phenotype of adipocytes have been previously described (8-10). This phenotypic variation may be due to differences in the progenitor populations from which new adipocytes are developed $(9,11)$. Of particular interest is a subset of adipocytes in the white adipose tissue of mice that arise de novo from an unconventional progenitor source, bone marrow-derived cells (BMDCs). These bone marrow derived cells of the hematopoietic myeloid lineage traffic through the circulation to the adipose tissue and commit to the adipocyte lineage in mice (12-16) and humans $(17,18)$.

Mouse studies have shown that bone marrow-derived adipocytes (BMDA) appear in a sex- and age-specific manner, with the greatest accumulation in the adipose tissue of older female mice (15). Interestingly, the regions that display the greatest accumulation of BMDA are those typically associated with metabolic dysfunction, the epi/pericardial and gonadal adipose depots $(15,16,18)$. Thus, we hypothesize that the suppression of gonadal hormone signaling regulates the development of BMDAs, resulting in their specific accumulation central adipose tissue depots.

Rodent models are often utilized in mechanistic studies of obesity. In mice, ovariectomy (OVX) is used as a model of the postmenopausal state, recapitulating the increase in body weight and, more importantly, the redistribution of body fat to central depots $(19,20)$ observed in postmenopausal women. Furthermore, estrogen receptor knockout models have convincingly demonstrated a role for estrogen in regulating fat mass (21-23). The observation that estrogen receptor alpha knockout $(\alpha E R K O)$ mice have a greater fat mass than WT mice suggests that $\mathrm{ER} \alpha$ plays a protective role against fat accumulation (21). In fact, Pedram et al. demonstrated that ER $\alpha$ plays a specific role in suppressing adipogenesis (24).

Based upon our previous findings that BMDA accumulation increases with age and studies demonstrating both OVX and $\alpha$ ERKO increase adiposity we utilized surgical OVX with or without the administration of exogenous $17 \beta$-estradiol $\left(\mathrm{E}_{2}\right) \mathrm{com}$ bined with lineage labeled bone marrow transplantation (BMT) models [e.g., green fluorescent protein (GFP) or luciferase] to determine the influence of gonadal hormone status on BMDA accumulation. We also utilized a whole-body $\alpha$ ERKO mouse model to interrogate disruptions in $\mathrm{E}_{2}$ signaling through $\mathrm{ER} \alpha$ as a mechanism by which ovarian hormones may regulate BMDA production. We hypothesized that OVX and $\alpha$ ERKO would result in increased production of BMDAs in metabolically detrimental depots (e.g., gonadal), and that this increase would be attenuated by estrogen replacement.

\section{MATERIALS AND METHODS}

\section{Animals}

All animal procedures were performed in an AAALACaccredited facility in accordance with the Guide for the Care and Use of Laboratory Animals (25) and approved by the
University of Colorado (CU) Denver Institutional Animal Care and Use Committee. Wild-type C57BL/6J mice from The Jackson Laboratory (\#000664) were used in all experiments unless otherwise stated. If applicable, OVX was performed by The Jackson Laboratory at 6 weeks of age, and all animals, male and female, arrived at $\mathrm{CU}$ at 8 weeks of age. Additional transgenic animals purchased from The Jackson Laboratory included estrogen receptor alpha null ( $\alpha$ ERKO) mice (\#004744), adiponectin promoter driven cre-recombinase (AdipoQ-cre) expressing mice (\#010803), adipocyte protein 2 driven crerecombinase (aP2-Cre) expressing mice (\#005069), and mice expressing enhanced GFP driven by the human ubiquitin-C promoter (\#004353). LoxP/stop/loxP (LSL) Luciferase mice were acquired from the National Cancer Institute's mouse repository (stock \#01XAC). Cre-recombinase and loxP expressing animals were bred in-house to create hemizygous cre and hemizygous lox expressing animals to use in future studies. Mice were maintained on a $12 \mathrm{~h}$ light, $12 \mathrm{~h}$ dark schedule at room temperature $\left(22^{\circ} \mathrm{C}\right)$ with ad libitum access to water and standard rodent chow free of phytoestrogens, Envigo Teklad global soy protein-free extruded diet (\#2920X), throughout all studies.

\section{Bone Marrow Transplantation}

Bone marrow donors were euthanized by isoflurane inhalation and cervical dislocation. Fresh BM cells were harvested aseptically from the femurs and tibias using a 27-gauge needle/syringe and $2 \mathrm{ml}$ phosphate-buffered saline. The cells were disaggregated by gentle pipetting several times and filtered to obtain the single cell suspension utilized in transplantation.

Male and female animals necessitating BMT for lineage tracing studies underwent transplantation after 1-2 weeks of acclimatization (at approximately 9-10 weeks of age). Recipient mice were irradiated with a 12 Gy total dose, split into 6 Gy doses, separated by $4 \mathrm{~h}$, using an X-ray radiation source. Immediately following the second dose, recipients were injected via the retroorbital venous plexus with $1 \times 10^{6} \mathrm{BM}$ cells suspended in $100 \mu \mathrm{l}$ sterile saline.

The BM used for transplantation came from one of the following transgenic mouse strains: AdipoQ-cre X LSL-Luciferase, aP2-cre X LSL-Luciferase or Ubc-GFP donors. AdipoQ-cre and aP2-cre mice use the adipocyte-specific adiponectin (AdipoQ) or fatty acid binding protein $4(\mathrm{aP} 2)$ gene promoters, respectively, to drive expression of cre-recombinase. The LSL-Luciferase mice carry a luciferase reporter gene downstream of a loxP-flanked stop codon. In cells expressing cre-recombinase, the stop codon is excised allowing expression of the luciferase reporter gene. Thus, BM from dual transgenic AdipoQ-cre x LSL-Luciferase or aP2cre x LSL-Luciferase mice has a luciferase reporter gene that is only expressed in mature adipocytes. When this BM is transplanted into WT mice, only mature adipocyte arising from the transplanted BM will express luciferase, identifying BMDAs. Ubc-GFP mice exhibit expression of GFP under control of the human ubiquitin- $\mathrm{C}$ promoter in all cell types throughout the body. Thus, all cells arising from BM transplanted from Ubc-GFP mice will be positive for GFP, including mature adipocytes of the BM lineage. 


\section{Hormone Replacement in Female Mice}

One to two weeks after BMT (at approximately 10-12 weeks of age, approximately 6 weeks after OVX), hormone replacement was initiated via implantation of subcutaneous pellets in those mice randomized to the $\mathrm{E}_{2}$ add-back group (Innovative Research of America, Estradiol: Cat. \# SE-121, 0.05 mg/pellet, 60-day release) or a sham surgery control group. Pellets were implanted in the dorsal subcutaneous tissue of the mouse. One $E_{2}$ pellet was introduced via 10-gauge trocar. Implants were expected to maintain circulating hormone levels in the physiological range with the doses previously utilized in other mouse studies of adipogenesis and $\mathrm{E}_{2}$ replacement after OVX (26).

\section{Whole-Body Luciferase Imaging}

Whole-body luciferase activity was completed with the IVIS Imaging System 50. Animals were lightly anesthetized and injected with $\mathrm{D}$-luciferin $(120 \mathrm{mg} / \mathrm{kg}, 100 \mu \mathrm{l}$ retro-orbital). Measurements were initiated $3 \mathrm{~min}$ after luciferin injection, and luminescence was integrated over $5 \mathrm{~min}$. In some animals, in vivo imaging was repeated at 2, 4, 6, 8, and 10 weeks posttransplant.

At the end of the study, mice were euthanized by $\mathrm{CO}_{2}$ asphyxiation and cervical dislocation. Body weight of the mice was measured immediately before sacrifice (18 weeks of age, 8-10 weeks posttransplant). Gonadal, inguinal, and interscapular (mix of white and brown fat) adipose depots and if appropriate, lower hind limb skeletal muscle (combined gastrocnemius and soleus), liver, and lung, were recovered, weighed, and processed for appropriate assay.

\section{Staining and Flow Cytometry}

Adipose tissue was minced into pieces of $1-3 \mathrm{~mm}$. Tissue fragments were digested at $37^{\circ} \mathrm{C}$ for $1 \mathrm{~h}$ with gentle shaking in digestion buffer [Krebs-Ringer HEPES $+2.5 \mathrm{mM}$ glucose $+2 \%$ fetal bovine serum (FBS) (Gemini Bio-products, \#100-500) + $200 \mathrm{nM}$ adenosine (Sigma Aldrich, \#A4036) + $1 \mathrm{mg} / \mathrm{ml}$ collagenase (type VIII, Sigma Aldrich, C2139), pH 7.4] using $4 \mathrm{ml}$ buffer/gram of fat. Samples were then passed through a $150 \mu \mathrm{m}$ Celltrics filter (Sysmex Partec GmbH, Germany, \#04-004-2324), and digestion stopped by the addition of one volume of wash buffer [Hanks Balanced Salt Solution (Mediatech, \#21-022-CV) + 2\% FBS + $200 \mathrm{nM}$ adenosine, $\mathrm{pH}$ 7.4]. Adipocytes were separated from the stromal pellet via centrifugation at $300 \mathrm{~g} 10 \mathrm{~min}$, and the wash step repeated to ensure sufficient separation and exclusion of digestion buffer. The adipocyte and stromal fractions were collected and prepared for appropriate experiments.

\section{Adipocyte Preparation}

Human TruStain FcX ${ }^{\mathrm{TM}}$ (Fc Receptor Blocking Solution, Cat. No. 422301; BioLegend, San Diego, CA, USA) was added to isolated adipocytes at $5 \mu \mathrm{l} / 100 \mu \mathrm{l}$ of cell suspension, and cells incubated at room temperature for $10 \mathrm{~min}$. LipidTOX Deep Red Neutral Lipid Stain (Cat. No. H34477; Life Technologies, Thermo Fisher Scientific Inc., Waltham, MA, USA, 1:200 dilution) and Vybrant ${ }^{\circledR}$ DyeCycle ${ }^{\mathrm{TM}}$ Violet stain (Invitrogen, Cat. \# V35003, $1 \mu \mathrm{l} / 10^{6}$ cells) were added to the cell suspension and incubated protected from light at $37^{\circ} \mathrm{C}$ for $30 \mathrm{~min}$. Cells were kept at $37^{\circ} \mathrm{C}$ until analysis. Cytosolic GFP expression was assessed in intact adipocytes
(LipidTOX Red ${ }^{\mathrm{POS}}$ events) containing a single nucleus (DyeCycle Violet $^{\mathrm{POS}}$ events) by flow cytometry as detailed previously.

\section{Stromal Cell Preparation}

The stromal cell pellet was resuspended in eBioscience 1X Red Blood Cell Lysis Buffer (\#00-4300-54) at room temperature for $5 \mathrm{~min}$. Stromal cells were pelleted by centrifugation at $500 \mathrm{~g}$ $5 \mathrm{~min}$ and washed by resuspension in $5 \mathrm{ml}$ wash buffer (HBSS with $1 \%$ FBS) followed by another centrifugation to pellet. Adipose stromal cells were stained with human TruStain FcX ${ }^{\mathrm{TM}}$ and antibodies to CD11b-PE (BD Biosciences, \#557397), CD45APC (BioLegend, \#103112), at $0.25 \mu \mathrm{g} / 10^{6}$ cells. Samples were incubated at $4^{\circ} \mathrm{C}$ in the dark for $25 \mathrm{~min}$. Following incubation samples were centrifuged to remove unbound antibodies, and the cells were resuspended in PBS containing 5\% FBS. GFP, $\mathrm{CD} 45$, and CD11b expression were assessed in single stromal cells according to our previously published flow cytometry gating strategy (27).

All cells were sorted at the University of Colorado Cancer Center Flow Cytometry Core facility using a MoFlo XDP cell sorter with Summit 4.3 software. The sheath fluid was IsoFlow, and the sample flow rate was set to a pressure differential of less than 0.4 psi. Sort mode was set to Purify 1. Appropriate signal compensation was set using single color and fluorescent minus one control samples.

\section{Adipose Tissue Depot Luciferase Assay}

At the end of the study, 10-12 weeks posttransplant, mice were euthanized immediately after whole-body imaging. Gonadal, inguinal, and interscapular fat pads along with lower hind limb skeletal muscle (combined gastrocnemius and soleus), liver, and lung were harvested and analyzed for organ-specific luminescence. IVIS imaging data were processed with Living Image 3.0 software. $100 \mathrm{mg}$ of each adipose tissue depot was homogenized, and cytosolic extracts were assayed with the Promega DualLuciferase Reporter Assay System (\#E1910).

\section{GFP DNA}

Adipocytes from mice that underwent BMT from transgenic mice in which GFP was ubiquitously expressed were isolated by collagenase digestion and centrifugation as described earlier. The freshly isolated adipocytes were immediately processed with the Qiagen DNA Micro kit (\#56304). Additional DNA was isolated from GFP transgenic or wild-type animals to be used for a control dilution series of known GFP DNA concentrations. Isolated DNA was quantified on a nano-drop ND1000 spectrophotometer, and subsequent PCR reactions were prepared at a standard concentration of $5 \mathrm{ng}$ genomic DNA per reaction. A housekeeping gene, Gapdh, was simultaneously quantified and used to normalize the GFP expression by $\Delta$ Ct. The percentage of GFP DNA was calculated from regression analysis of the control dilution series $\Delta \mathrm{Ct}$ values. $\mathrm{qPCR}$ reactions were prepared using the Thermo DyNAmo Flash SYBR Green qPCR kit (\#F415) according to manufacturer's directions with primers diluted to a final concentration of $0.5 \mu \mathrm{M}$ per reaction. $\mathrm{qPCR}$ primers were purchased from Integrated DNA Technologies and are as follows (listed as $5^{\prime}-3^{\prime}$ ) Gapdh forward: TAC GCA TTA TGC CCG AGG 
AC, Gapdh reverse: TGT AGG CCA GGT GAT GCA AG, GFP forward: CCA CAT GAA GCA GCA GGA CTT, GFP reverse: GGT GCG CTC CTG GAC GTA. qPCR reactions were run in triplicate on an Applied Biosystems StepOne real-time PCR system.

\section{Statistical Analysis}

Between-group comparisons were evaluated using one-way (body weight and circulating factors) or two-way (depot weight, luciferase, and GFP measurements; group $\times$ depot) ANOVA. When indicated by a significant $F$-statistic, post hoc-analyses to determine significant mean differences between the groups were conducted with the Bonferroni or Dunnett multiple comparison tests (adjusted $p$-values presented). All results are presented as mean \pm SEM unless otherwise noted, and $\alpha$ was set at 0.05 . Statistical analysis was completed in GraphPad Prism v7.03.

\section{RESULTS}

\section{Sex Difference in Accumulation of BM-Derived Adipocytes}

To determine whether BMDA production is higher in female or male mice, cytosolic GFP expression was measured in intact adipocytes (LipidTOX Red ${ }^{\mathrm{POS}}$ events) containing a single nucleus (DyeCycle Violet ${ }^{\text {POS }}$ events) by flow cytometry as an indicator that the adipocytes arose from a $\mathrm{BM}$ origin. The data show greater production of GFP ${ }^{\text {POS }}$ BMDA (green events enclosed in ovals) in both gonadal and inguinal adipose depots of female rather than male mice (Figure 1A). Measurement of whole-body luciferase activity (light emission) in mice that were transplanted with BM from which expression of a luciferase reporter gene was under the control of the adipocyte-specific adiponectin gene promoter demonstrated the same sex difference in production of BMDAs as indicated by greater whole-body light emission in female mice (Figure 1B). Luciferase activity in lysates from individual adipose tissue depots revealed higher luciferase activity in depots from female compared with male mice (main effect of $\operatorname{sex} p<0.01$ ), although only the difference in the gonadal depot reached statistical significance $(p=0.005$, Figure 1C). Luciferase activity was essentially absent in non-adipose tissues. Thus, at least in reproductively capable animals, female mice produce more BMDAs than male mice.

\section{Body and Adipose Tissue Depot Weights}

To investigate how ovarian hormones regulate the production of BMDA only female animals were included in all studies from here forward. To generate groups with different ovarian hormone levels we used WT or OVX mice with or without exogenous $\mathrm{E}_{2}$. Because $\mathrm{ER} \alpha$ is necessary for estrogen to suppress adipogenesis (24), we also utilized whole-body $\alpha$ ERKO mice.

As expected, by 18 weeks of age ( 8 weeks post-BMT and after 6 weeks of $\mathrm{E}_{2} / \mathrm{P}_{4}$ add-back) there was a significant difference in body weight between the groups (main effect of group, $p=0.0002$; Figure 2A). OVX mice were heavier than WT $(p=0.0001)$. Body weight was not different from WT in any other group. Similarly,
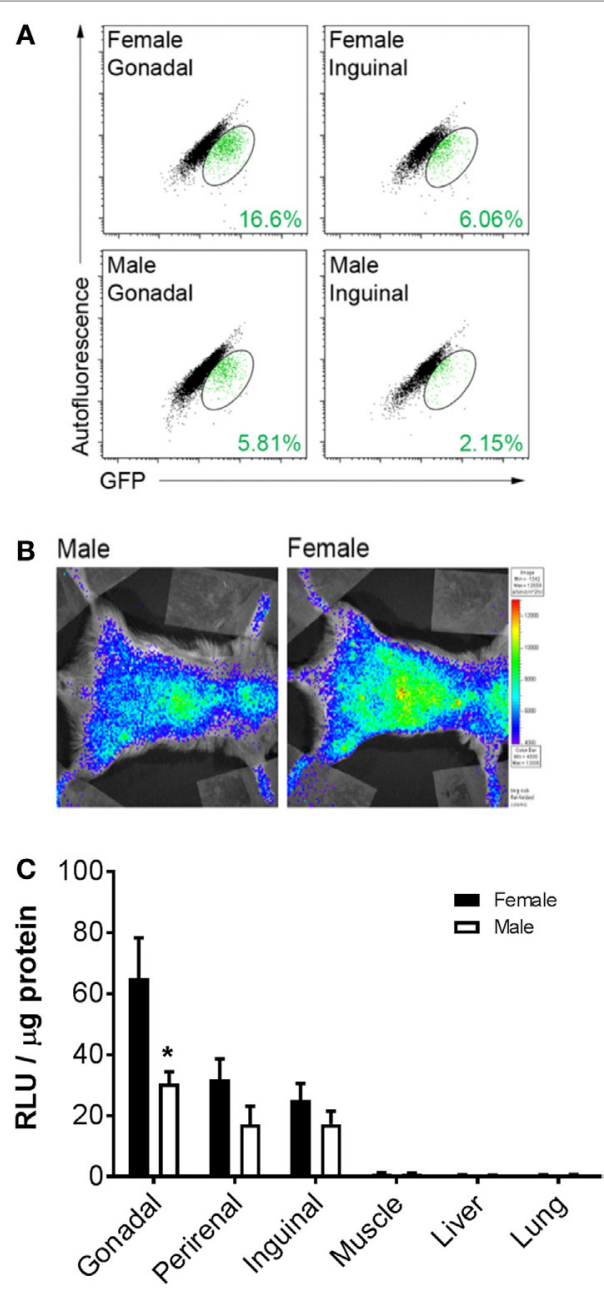

FIGURE 1 | Bone marrow-derived adipocyte (BMDA) production is higher in female rather than age-matched male mice. (A) Eight-week-old wild-type male and female mice underwent bone marrow transplantation from sex-matched transgenic mice in which green fluorescent protein (GFP) was ubiquitously expressed. After 12 weeks, gonadal and inguinal fat depots were harvested. Cytosolic GFP expression was assessed in intact adipocytes (LipidTOX RedPOS events) containing a single nucleus (dyecycle violetPos events) by flow cytometry. The percentage of BMDAs is denoted in green font in the lower right-hand corner of each scattergram. (B) Eight-week-old male and female wild-type mice underwent transplantation with BM from mice in which expression of a luciferase reporter gene was under the control of the adipocyte-specific adiponectin gene promoter (AdipoQ-Iuciferase donor mice). Twelve weeks posttransplant, whole-body luciferase activity (light emission) was measured in the recipients. Body-wide luciferase activity was consistently higher in female rather than male mice as shown by the representative images. (C) Adipose depots (gonadal, perirenal, and inguinal) and non-adipose depots (muscle, liver, and lung) were harvested from male and female AdipoQ-creLSL-Luciferase mice 12 weeks after transplant $(n=3$ for each sex). Luciferase activity in lysates from each tissue revealed higher luciferase activity in adipose tissue from female than male mice. ${ }^{*} p<0.05$ vs female of same depot. Data presented as mean \pm SEM.

OVX mice had larger, and OVX $+\mathrm{E}_{2}$ mice smaller, gonadal fat pads than WT ( $p=0.02$ and $p=0.0001$, respectively; Figure 2B). Estrogen replacement also resulted in a smaller interscapular fat 

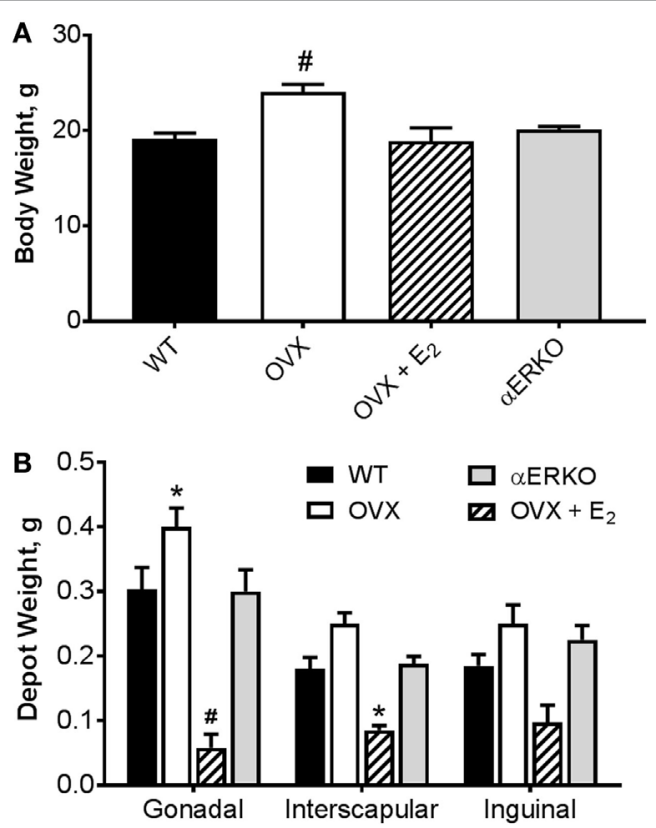

FIGURE 2 | Ovariectomy-induced increases body weight and adipose tissue depot weight are prevented with estradiol replacement. (A) Body weight was greater in OVX compared with WT mice. ${ }^{*} p<0.001$ vs WT [WT $n=14$, OVX $n=7, \mathrm{OVX}+\mathrm{E}_{2} n=5$, estrogen receptor alpha knockout $\left.(\alpha \mathrm{ERKO}) n=6\right]$. (B) Gonadal depot weight was higher in OVX and lower in OVX $+E_{2}$ mice compared with WT. Interscapular fat depot weight was lower in OVX $+E_{2}$ mice compared with WT. Inguinal depot weights were not different from WT in any group. ${ }^{*} p<0.05$ or ${ }^{*} p<0.001$ vs WT in same depot $\mathrm{WT} n=10$, OVX $\left.n=6, \mathrm{OVX}+\mathrm{E}_{2} n=4, \alpha \mathrm{ERKO} n=5\right)$. Data presented as mean $\pm \mathrm{SEM}$

pad compared with WT (Figure 2B). There were no differences between the groups in the inguinal depot.

\section{Production of BMDA With Sex Hormone Manipulation}

We measured light emission at the whole body and adipose tissue depot levels to determine if increases in whole body and fat pad weight could be resultant to the production of new BMDAs. By 6-weeks post-BMT, OVX and $\alpha$ ERKO mice had significantly more BMDAs compared with WT $(\mathrm{OVX} p=0.05$, $\alpha$ ERKO $p=0.003)$ as indicated by whole-body light emission (Figure 3B). At eight-weeks post-BMT, OVX and $\alpha$ ERKO mice still had more BMDAs ( $p=0.0002$ and $p=0.0013$, respectively), but OVX $+E_{2}$ mice also had fewer $(p=0.04)$, BMDAs compared with WT (Figures 3A,B). At the adipose tissue depot level, on average, luciferase activity was lowest in the interscapular depot (main effect of depot $p<0.0001$, Figure 3C). Consistent with the whole-body luciferase results, gonadal adipose tissue from OVX and $\alpha E R K O$ mice and inguinal adipose tissue from $\alpha$ ERKO mice had more BMDAs than WT (all $p<0.01$ ). Once again, estrogen replacement completely attenuated, and even decreased, production below WT levels in the gonadal depot ( $p=0.006$ vs WT).

As a complementary measurement to the adipocyte-specific luciferase model, we also utilized a model in which BM from
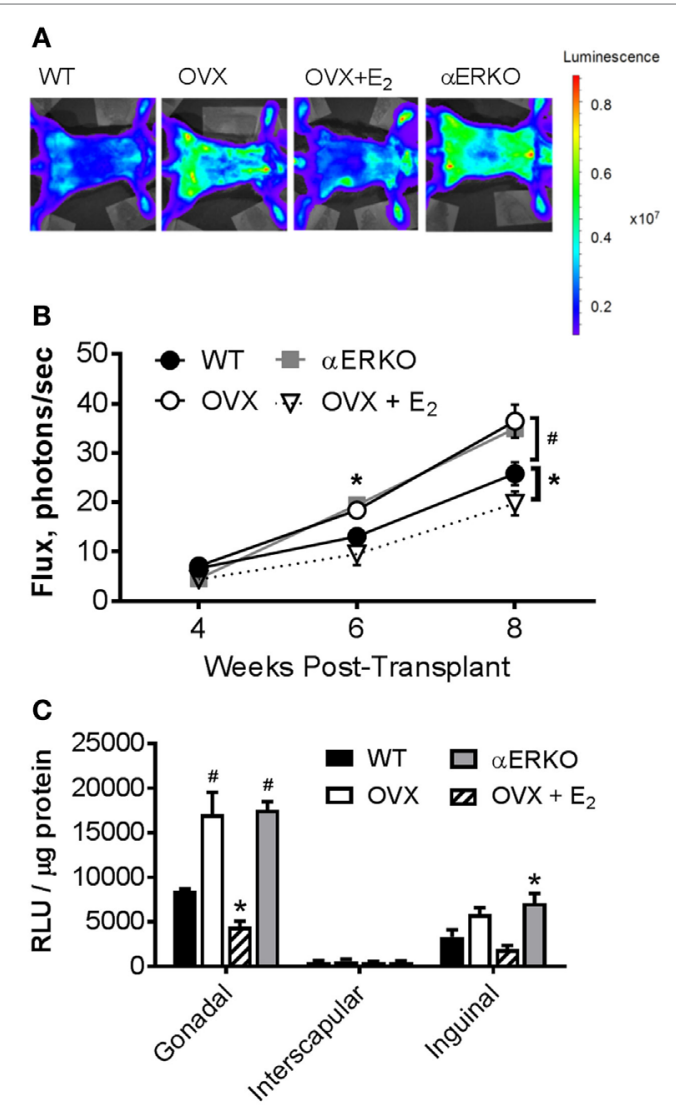

FIGURE 3 | Ovariectomy increases production of bone marrow-derived adipocytes (BMDA) in mice as determined by aP2-cre X LoxP/stop/ loxP-Luciferase lineage quantitation. Bone marrow transplantation from donors in which luciferase expression was guided by the fatty acid binding protein 4 (also called adipocyte protein 2 or aP2) gene promoter (aP2luciferase donor mice) was performed at 8 weeks of age in wild-type recipient mice. Luciferase activity (light emission in the wild-type recipient mice) indicates production of BM-derived adipocytes. (A) Representative images of whole-body light emission. (B) In vivo whole-body light emission measurements were completed 4, 6, and 8 weeks posttransplant. ${ }^{*} p<0.05$ and ${ }^{*} p \leq 0.001$ vs WT at same time point $(\mathrm{WT} n=4$, all other groups $n=3$ ). (C) Immediately after the last whole-body imaging measurement the mice were euthanized, and gonadal, interscapular and, inguinal fat pads harvested and analyzed for luminescence [expressed as relative light units $(\mathrm{RLU})]$. ${ }^{*} p<0.05$ and ${ }^{*} p=0.0001$ vs WT in each depot; $\mathrm{WT} n=4$, all other groups $n=3$. Data presented as mean \pm SEM.

transgenic mice in which GFP was ubiquitously expressed was transplanted into WT or OVX mice, some with $\mathrm{E}_{2}$ replacement. In this model, the GFP DNA content in isolated adipocytes from specific adipose tissue depots (Figure 4A) or the percentage of $\mathrm{GFP}^{\mathrm{POS}}$ adipocytes measured by flow cytometry (Figure $4 \mathrm{~B}$ ) was indicative of BMDA production. As described in detail previously (27), BMDA progenitor cell accumulation was quantified by the percentage of GFP ${ }^{\text {IM }}$ cells present in the adipose tissue stroma (Figure 4C). Consistent with the luciferase model, BMDA production was the greatest in the gonadal depot (main effect of depot, $p<0.0001$ for GFP DNA and $p=0.004$ for GFP ${ }^{\text {POs }}$ adipocytes by flow; Figures 4A,B). Gonadal fat from OVX mice demonstrated the greatest production of BMDAs ( $p=0.004$ vs 

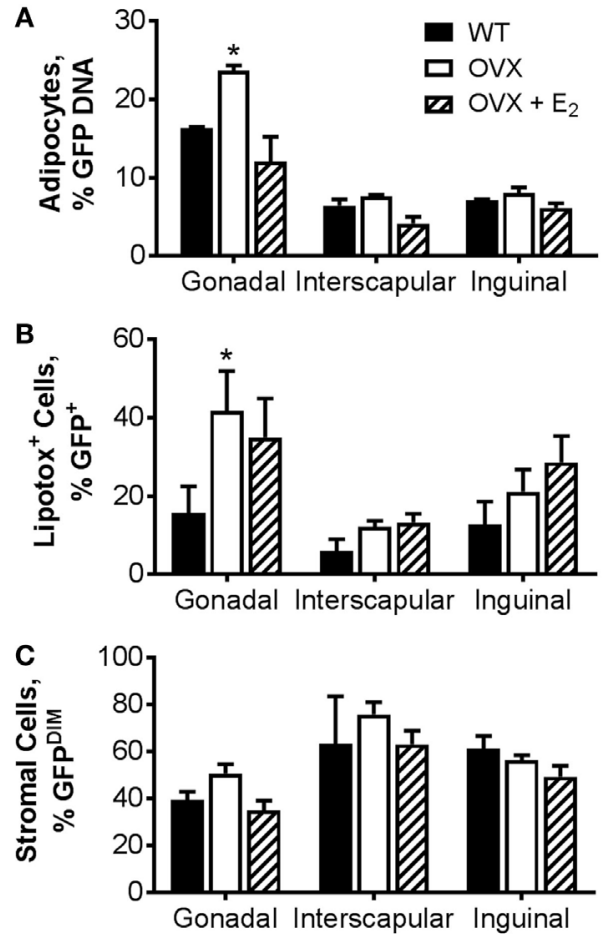

FIGURE 4 | Ovariectomy increases production of bone marrow-derived adipocytes (BMDAs) as determined by green fluorescent protein (GFP) lineage labeling. Eight-week-old WT or OVX mice underwent bone marrow transplantation from transgenic mice in which GFP was ubiquitously expressed. After 2 weeks, a subset of OVX animals was randomized to receive $\mathrm{E}_{2}$ replacement (all $n=3$ ). After 8 weeks, mice were euthanized, and adipose tissue from gonadal, interscapular, and inguinal fat depots harvested. (A) The percent of floated adipocytes cells containing GFP DNA normalized for Gapdh was quantified in floated adipocytes from each depot. (B) Percentage of intact adipocytes that were GFP+ (BMDAs) as analyzed by flow cytometry. (C) Percentage of stromal cells that were GFPDIM (representing bone marrow-derived cells) as analyzed by flow cytometry. ${ }^{*} p<0.05$ vs WT in same depot. Data presented as mean \pm SEM.

WT for GFP DNA and $p=0.02$ for flow), which was once again prevented with estrogen replacement. The sex hormone intervention did not appear to have a significant effect on accumulation of BMDA progenitor accumulation in any depot (Figure 4C).

\section{DISCUSSION}

Here, we demonstrate for the first time that ovarian hormone status in female mice regulates the production of BMDAs. Utilizing multiple transgenic fate mapping models, we observed that surgical depletion of ovarian hormones post-ovariectomy augmented the production of BMDAs in the gonadal depot. Furthermore, estradiol replacement consistently attenuated the accelerated production of BMDAs. In addition, genetic knockdown of ER $\alpha$ also enhanced BMDA production in both the gonadal and inguinal depots. Taken together, our data suggest that estradiol regulates $\mathrm{BMDA}$ production.

The mechanisms underlying the shift toward preferential abdominal adiposity with decreased circulating ovarian hormones in both women and female mice are not completely understood. Importantly, increased central adiposity is associated with elevated risk for cardiovascular disease (28), a leading cause of death in postmenopausal women. Thus, continued pursuit of uncovering the mechanisms underlying this phenomenon in women over the menopausal transition is critical in maintaining women's health across the lifespan.

Central adiposity may be imposing detrimental health effects not only through mechanisms associated with increased adipose tissue volume but also changes in its adipocyte composition. Although non-adipocyte cell accumulation, local blood flow and neural innervation are important determinants of the local adipose tissue microenvironment, inherent characteristics of the constituent adipocytes differ by depot. Importantly, these depot-specific characteristics are retained even when the cells are studied in vitro (e.g., adiponectin secretion, insulin action, and signaling) $(8-10,29)$. Recently, alternatives to the traditional adipose tissue resident mesenchymal lineage origin for adipocytes have been proposed (e.g., BM progenitors or BMDCs) (30). Thus, adipocytes arising from varying lineages of multipotent mesenchymal progenitors $(9,11,31,32)$ and hematopoietic progenitors (15) may contribute to differences in adipocyte characteristics and phenotypes both between and within adipose tissue depots. However, in adults, the initiating or limiting factors for the production of new adipocytes from varying lineages remain uncertain. Better understanding of these mechanisms may uncover new targets for novel health and weight management strategies.

\section{Sex Differences}

The BMDA is a novel "alternative lineage" adipocyte, a likely candidate in the regulation of adipose tissue heterogeneity. We previously observed a sex difference in the production of BMDAs in male and female mice, confirmed herein, with female mice producing more BMDAs compared with males (30). Although observed in all major fat depots, the highest production of BMDAs is evident in gonadal fat, considered a visceral fat depot in mice. Global gene expression analysis reveals a detrimental metabolic phenotype characterized by inflammatory and mitochondrial related genes divergently expressed between conventional white and BMDAs (15), further supporting the contribution to depot heterogeneity.

The greater production of BMDAs in young female compared with male mice appears counterintuitive. However, females have higher total and percent body fat compared with males. Thus, females may have more BMDAs throughout life resultant to their greater relative fat mass. In addition, the rise in plasma estradiol at puberty and the decline with menopause are both associated with increased fat mass in women. A similar dual-phase relationship may exist with estradiol regulation of BMDA production in female mice. Whether the sex difference observed in mice translates to humans is unknown.

\section{Alterations in Cellular Composition}

Animal models are ideal for exploring the relationship between $\mathrm{E}_{2}$ and the adipocyte. Alterations in the production of new adipocytes (adipogenesis) as well as adipocyte size occur with 
the loss of circulating ovarian hormones in mice. For example, ovariectomy leads to an increase in adipocyte size, along with increases in overall body fat and visceral adiposity, which is fully preventable by $\mathrm{E}_{2}$ administration (20). Similarly, the aromatase knockout mouse, which is unable to synthesize endogenous estrogens, has increased body weight and visceral adiposity compared with WT animals $(33,34) . \mathrm{E}_{2}$ replacement prevents this increase in body weight and central adiposity and decreases adipocyte size $(33,34)$. Finally, $\alpha$ ERKO mice exhibit increased adiposity, particularly in the visceral depots accompanied by increases in adipocyte volume and number (21). Importantly, exogenous estrogen therapy in these mice is not effective at reducing fat mass (23). Thus, it is clear that estrogen status, and signaling through ER $\alpha$ in particular, has an important effect on adipose tissue volume and cellularity. However, if the "type" of adipocytes primarily produced or altered in the estrogendeficient state are the same as those in the estrogen replete state is unknown.

Unlike previous studies, compared with WT we did not observe elevated fat mass in our $\alpha E R K O$ mice. This could be due to the fact that irradiated animals and/or animals maintained at Aurora, CO, USA, altitude $(1,600 \mathrm{~m})$ eat less, gain less weight, and exhibit decreased adiposity compared with unirradiated animals maintained at lower altitudes (12). On the other hand, we did observe the expected OVX-induced increase in body weight and adiposity. This difference between models may have been a result of the relatively short timeline (8-10 weeks after transplant) over which the study was conducted. Within 15 days, OVX mice already have a higher body weight compared with sham controls (35), while differences in body weight in $\alpha$ ERKO are not reported until 12-16 weeks $(21,36-39)$. Importantly, our results suggest that weight gain is not a prerequisite for the production of BMDAs, at least resultant to disrupted estrogen signaling. Whether a preferential increase in the production of BMDAs compared with conventional lineage adipocytes is responsible for the observed OVX-induced weight gain cannot be determined from the methods utilized in this study. However, studies investigating the turnover rate of both lineages of adipocytes are critical to answer this question.

Both Vieira Potter et al. (40) and Rogers et al. (19) observed increased macrophage infiltration and activation in gonadal adipose tissue as early as 12 weeks post-OVX. Notably, BMDA appear to develop via a novel transdifferentiation of adipose tissue macrophages (27), and we consistently observe their highest production in the gonadal fat depots $(15,18)$. However, we did not observe a difference in the percentage of BMDA progenitors (i.e., GFP ${ }^{\mathrm{DIM}}$ cells) in adipose stroma between WT and the OVX, OVX $+\mathrm{E}_{2}$ or $\alpha \mathrm{ERKO}$ groups. Similar results were obtained in mice treated with high fat diet or rosiglitazone, both of which exhibited more mature BMDAs without altered accumulation BM-derived progenitors in the SVF (12). Of note, we did not quantify the proportion of different SVF cell types (i.e., macrophage, $\mathrm{T}$ and $\mathrm{B}$ lymphocytes, neutrophils, eosinophils, dendritic cells, endothelial cells, fibroblasts, and mesenchymal stem cells) or the activation state of resident macrophages. Either of those measurements could reveal alterations at the SVF level that were not detected with our generalized GFP quantification of a heterogeneous population of stromal cells.

Our findings suggest that changes in ovarian hormone status/ signaling result in alterations in the cellular composition of adipose tissue that may not contribute to alterations in the size of the adipose tissue depot. Notably, previous research indicates that obesity per se is not required for the development of menopauseassociated metabolic disturbances $(40,41)$. Thus, future studies are warranted to investigate the relationship between weight gain/energy balance, rate of production of BMDAs and declines in metabolic health after OVX in female mice.

\section{Role for Estradiol in BMDA Production}

Our results consistently demonstrated that estradiol replacement successfully attenuates the production of BMDAs as well as the OVX-induced increase in body weight. It appears as though $\mathrm{E}_{2}$ signaling through $\mathrm{ER} \alpha$ is one mechanism by which estrogen may regulate the production of BMDAs. These studies complement existing evidence that estrogen has potentially beneficial effects on the adipose tissue through ER $\alpha$ signaling. ER $\alpha$ has been identified has having important roles in maintaining not only adipocyte size and number but also adipose tissue inflammation and fibrosis, as demonstrated in adipose tissue knockout and gonadal depot-specific ER $\alpha$ knockdown (42). Interestingly, mouse models specifically knocking out $\mathrm{ER} \alpha$ in hematopoietic or myeloid lineage cells, or transplanting $\mathrm{ER} \alpha^{-/-} \mathrm{BM}$ into LDL Receptor knockout mice, all result in increased adipose tissue mass (43). The additional adipose tissue in these mice is accompanied by increased adipocyte size, number of crown like structures, chemokine expression, and immune cell infiltration and inflammation in the gonadal adipose tissue. Increased accumulation of BMDCs in the adipose tissue of these mice, along with their observed insulin resistance, demonstrates the importance of myeloid cell ER $\alpha$ expression in the maintenance of adipose tissue homeostasis (43).

It is also possible that the removal of $\mathrm{ER} \alpha$ in our model resulted in increased BMDA accumulation through greater ER $\beta$ signaling. Because of disrupted negative feedback regulation, circulating estrogen levels can be higher in $\alpha$ ERKO than WT $(22,44)$, and the increase in fat mass observed in $\alpha E R K O$ mice is attenuated with OVX, suggesting that estrogen signaling through ER $\beta$ plays at least some part in the elevated fat mass evident in $\alpha E R K O$ mice (45). Because we did not perform OVX in our $\alpha E R K O$ mice we cannot rule out a role for $\operatorname{ER} \beta$ in the increased production of BMDAs. However, our consistent results between the OVX (low circulating estrogens and intact $\mathrm{ER} \alpha$ ) and $\alpha \mathrm{ERKO}$ (high circulating estrogens and low ER $\alpha$ ) models suggest reduced signaling through $\mathrm{ER} \alpha$ does play a role. Future studies designed specifically to isolate the roles of $\mathrm{ER} \alpha, \mathrm{ER} \beta$, and the $\mathrm{G}$ protein-coupled estrogen receptor (GPER or GPR30) are critical in the complete understanding of the role of $\mathrm{E}_{2}$ in production of BMDAs.

\section{Limitations}

While OVX is often used as a rodent model of menopause, the surgical intervention results in a rapid decline in ovarian hormones and eliminates the perimenopausal period when women experience fluctuations in hormone levels and irregular menstrual 
cycles. Although a very useful model for basic mechanistic studies, future studies utilizing the chemical 4-vinylcyclohexene diepoxide to mimic a more prolonged variable menopausal transition will provide a translation to the perimenopausal period. Furthermore, the mice were ovariectomized at 8 weeks of age, which, although not uncommon, does not recapitulate the older age at which women would usually undergo natural (or even surgical) menopause. In addition, OVX was conducted before the BMT. It is possible that OVX occurring at a later age, or after the BMT, would alter our findings.

Although the effects of OVX and $\mathrm{E}_{2}$ add-back on BMDA production were only statistically significant in the gonadal depot, the inguinal depot tended to respond similarly. It is possible that the lower overall BMDA production in the inguinal depot as well as the small sample size limited our statistical power to detect significant differences between the groups in this depot. Continuing to study the region-specific production of BMDAs will be important in future studies.

The $\mathrm{E}_{2}$ add-back regimen utilized $0.05 \mathrm{mg}$ 60-day constant release pellets. This was expected to maintain circulating estrogen levels within the physiological range. However, a limitation of this study is that we do not have the samples available to measure circulating $\mathrm{E}_{2}$ in our mice to confirm the actual values. Our observation that gonadal adipose tissue weight was lower in the $\mathrm{OVX}+\mathrm{E}_{2}$ compared with WT suggests the $\mathrm{E}_{2}$ replacement could have been supraphysiologic. However, whole body or inguinal adipose depot weights were not statistically different between the WT and $\mathrm{OVX}+\mathrm{E}_{2}$ groups. Furthermore, previous literature reports that higher dose $\mathrm{E}_{2}$ pellets (e.g., 0.25 or $0.1 \mathrm{mg}$ ) result in circulating $\mathrm{E}_{2}$ levels well within physiological range $(<117-734 \mathrm{pmol} / \mathrm{l})(46,47)$ therefore it is unlikely, although not impossible, that our low dose resulted in supraphysiological replacement. In addition, whether a constant release single hormone add-back regimen leads to different results than a more physiological cyclical combination hormone replacement is uncertain.

The whole-body $\alpha$ ERKO mice used in this study carried the $E s r 1^{\text {tmlKsk }}$ allele. These mice express a low level of a truncated form of $\mathrm{ER} \alpha$ due to alternate splicing of the transcript resulting in a small amount of estrogen binding $(\sim 3 \%)$ in the uterine tissues of the homozygote animals as compared with wild-type controls. Importantly, no $\mathrm{E}_{2}$ binding was detected in other tissues of this strain of animal (e.g., brain, kidney, and liver) (The Jackson Lab, Stock No. 004744; https://www.jax.org/strain/004744). Recently, Fatima and colleagues reported estrogen receptor 1 (Esr 1 or ER $\alpha)$ mRNA expression is significantly lower in gonadal and inguinal adipose tissue from this same strain of ERKO mice compared with C57BL WT controls (48). However, because measurement of BMDA production required use of the entire fat pad, we did not confirm the lower the Esr1 gene expression in the adipose tissue of the mice studied here.

\section{REFERENCES}

1. Popkin BM, Doak CM. The obesity epidemic is a worldwide phenomenon. Nutr Rev (1998) 56(4 Pt 1):106-14. doi:10.1111/j.1753-4887.1998.tb01722.x

2. Flegal KM, Kruszon-Moran D, Carroll MD, Fryar CD, Ogden CL. Trends in obesity among adults in the United States, 2005 to 2014. JAMA (2016) 315(21):2284-91. doi:10.1001/jama.2016.6458

\section{CONCLUSION}

Our results suggest that, in female mice, the loss of ovarian hormone results in increased production of BMDAs. In particular, the loss of estradiol, possibly through $\mathrm{ER} \alpha$ appears to be important in regulating the production of de novo BMDAs. Thus, a shift in the adipocyte composition of adipose tissue depots toward more BMDA production with the loss of ovarian hormones in females could be related to the increased metabolic disease risk observed in this population. Studies to investigate the metabolic phenotype and physiological impact of the production of BMDAs are needed before a direct mechanistic link can be confirmed.

\section{DATA AVAILABILITY STATEMENT}

The raw data supporting the conclusions of this manuscript will be made available by the authors, without undue reservation, to any qualified researcher.

\section{ETHICS STATEMENT}

All animal procedures were performed in an AAALAC-accredited facility in accordance with the Guide for the Care and Use of Laboratory Animals (25) and approved by the University of Colorado Denver Institutional Animal Care and Use Committee.

\section{AUTHOR CONTRIBUTIONS}

DK, SM, and WK contributed to conception and design of the study; TS and DK performed data collection; KG and DK performed data analysis; KG, DK, SM, and WK performed data interpretation; $\mathrm{KG}$ wrote the first draft of the manuscript; $\mathrm{KG}$, TS, and DK wrote sections of the manuscript. All the authors contributed to manuscript revision, read and approved the submitted version.

\section{ACKNOWLEDGMENTS}

We would like to thank Heidi Miller and Paul Erickson for their technical expertise and contributions to this project.

\section{FUNDING}

This work was supported by the National Institutes of Health [R01 DK078966 (to DK), P50 HD073063 (to WK), K01 DK109053 (to KG), and P30 CA046934], the University of Colorado Center for Women's Health Research, and the Eastern Colorado VA Geriatric Research, Education, and Clinical Center. The contents do not represent the views of the U.S. Department of Veterans Affairs or the United States Government.

3. Bjorntorp P. Body fat distribution, insulin resistance, and metabolic diseases. Nutrition (1997) 13(9):795-803. doi:10.1016/S0899-9007(97)00191-3

4. Wajchenberg BL. Subcutaneous and visceral adipose tissue: their relation to the metabolic syndrome. Endocr Rev (2000) 21(6):697-738. doi:10.1210/ edrv.21.6.0415

5. Carr MC. The emergence of the metabolic syndrome with menopause. J Clin Endocrinol Metab (2003) 88(6):2404-11. doi:10.1210/jc.2003-030242 
6. Abdulnour J, Doucet E, Brochu M, Lavoie JM, Strychar I, Rabasa-Lhoret R, et al. The effect of the menopausal transition on body composition and cardiometabolic risk factors: a Montreal-Ottawa New Emerging Team group study. Menopause (2012) 19(7):760-7. doi:10.1097/gme.0b013e318240f6f3

7. Karvonen-Gutierrez C, Kim C. Association of mid-life changes in body size, body composition and obesity status with the menopausal transition. Healthcare (Basel) (2016) 4(3):42. doi:10.3390/healthcare 4030042

8. Tchkonia T, Giorgadze N, Pirtskhalava T, Tchoukalova Y, Karagiannides I, Forse RA, et al. Fat depot origin affects adipogenesis in primary cultured and cloned human preadipocytes. Am J Physiol Regul Integr Comp Physiol (2002) 282(5):R1286-96. doi:10.1152/ajpregu.00653.2001

9. Tchkonia T, Giorgadze N, Pirtskhalava T, Thomou T, DePonte M, Koo A, et al. Fat depot-specific characteristics are retained in strains derived from single human preadipocytes. Diabetes (2006) 55(9):2571-8. doi:10.2337/db06-0540

10. Perrini S, Laviola L, Cignarelli A, Melchiorre M, De Stefano F, Caccioppoli C, et al. Fat depot-related differences in gene expression, adiponectin secretion, and insulin action and signalling in human adipocytes differentiated in vitro from precursor stromal cells. Diabetologia (2008) 51(1):155-64. doi:10.1007/ s00125-007-0841-7

11. Tchkonia T, Lenburg M, Thomou T, Giorgadze N, Frampton G, Pirtskhalava T, et al. Identification of depot-specific human fat cell progenitors through distinct expression profiles and developmental gene patterns. Am J Physiol Endocrinol Metab (2007) 292(1):E298-307. doi:10.1152/ajpendo.00202. 2006

12. Crossno JT Jr, Majka SM, Grazia T, Gill RG, Klemm DJ. Rosiglitazone promotes development of a novel adipocyte population from bone marrow-derived circulating progenitor cells. J Clin Invest (2006) 116(12):3220-8. doi:10.1172/ JCI28510

13. Tomiyama K, Murase N, Stolz DB, Toyokawa H, O’Donnell DR, Smith DM, et al. Characterization of transplanted green fluorescent protein+ bone marrow cells into adipose tissue. Stem Cells (2008) 26(2):330-8. doi:10.1634/ stemcells.2007-0567

14. Sera Y, LaRue AC, Moussa O, Mehrotra M, Duncan JD, Williams CR, et al. Hematopoietic stem cell origin of adipocytes. Exp Hematol (2009) 37(9): 1108-20, 1120.e1-4. doi:10.1016/j.exphem.2009.06.008

15. Majka SM, Fox KE, Psilas JC, Helm KM, Childs CR, Acosta AS, et al. De novo generation of white adipocytes from the myeloid lineage via mesenchymal intermediates is age, adipose depot, and gender specific. Proc Natl Acad Sci U S A (2010) 107(33):14781-6. doi:10.1073/pnas.1003512107

16. Majka SM, Miller HL, Sullivan T, Erickson PF, Kong R, Weiser-Evans M, et al. Adipose lineage specification of bone marrow-derived myeloid cells. Adipocyte (2012) 1(4):215-29. doi:10.4161/adip.21496

17. Ryden M, Uzunel M, Hard JL, Borgstrom E, Mold JE, Arner E, et al. Transplanted bone marrow-derived cells contribute to human adipogenesis. Cell Metab (2015) 22(3):408-17. doi:10.1016/j.cmet.2015.06.011

18. Gavin KM, Gutman JA, Kohrt WM, Wei Q, Shea KL, Miller HL, et al. De novo generation of adipocytes from circulating progenitor cells in mouse and human adipose tissue. FASEB J (2016) 30(3):1096-108. doi:10.1096/fj. $15-278994$

19. Rogers NH, Perfield JW II, Strissel KJ, Obin MS, Greenberg AS. Reduced energy expenditure and increased inflammation are early events in the development of ovariectomy-induced obesity. Endocrinology (2009) 150(5):2161-8. doi:10.1210/en.2008-1405

20. Stubbins RE, Holcomb VB, Hong J, Nunez NP. Estrogen modulates abdominal adiposity and protects female mice from obesity and impaired glucose tolerance. Eur J Nutr (2012) 51(7):861-70. doi:10.1007/s00394-011-0266-4

21. Heine PA, Taylor JA, Iwamoto GA, Lubahn DB, Cooke PS. Increased adipose tissue in male and female estrogen receptor-alpha knockout mice. Proc Natl Acad Sci U S A (2000) 97(23):12729-34. doi:10.1073/pnas.97.23.12729

22. Lindberg MK, Alatalo SL, Halleen JM, Mohan S, Gustafsson JA, Ohlsson C. Estrogen receptor specificity in the regulation of the skeleton in female mice. J Endocrinol (2001) 171(2):229-36. doi:10.1677/joe.0.1710229

23. Lindberg MK, Weihua Z, Andersson N, Moverare S, Gao H, Vidal O, et al. Estrogen receptor specificity for the effects of estrogen in ovariectomized mice. J Endocrinol (2002) 174(2):167-78. doi:10.1677/joe.0.1740167

24. Pedram A, Razandi M, Blumberg B, Levin ER. Membrane and nuclear estrogen receptor alpha collaborate to suppress adipogenesis but not triglyceride content. FASEB J (2016) 30(1):230-40. doi:10.1096/f.15-274878
25. National Research Council (U.S.). Committee for the Update of the Guide for the Care and Use of Laboratory Animals, Institute for Laboratory Animal Research (U.S.), and National Academies Press (U.S.). Guide for the Care and Use of Laboratory Animals. 8th ed. Washington, DC: National Academies Press (2011)

26. Jeong S, Yoon M. 17beta-estradiol inhibition of PPARgamma-induced adipogenesis and adipocyte-specific gene expression. Acta Pharmacol Sin (2011) 32(2):230-8. doi:10.1038/aps.2010.198

27. Gavin KM, Majka SM, Kohrt WM, Miller HL, Sullivan TM, Klemm DJ. Hematopoietic-to-mesenchymal transition of adipose tissue macrophages is regulated by integrin betal and fabricated fibrin matrices. Adipocyte (2017) 6(3):234-49. doi:10.1080/21623945.2017.1314403

28. Cornier MA, Despres JP, Davis N, Grossniklaus DA, Klein S, Lamarche B, et al. Assessing adiposity: a scientific statement from the American Heart Association. Circulation (2011) 124(18):1996-2019. doi:10.1161/CIR. 0b013e318233bc6a

29. Perrini S, Cignarelli A, Ficarella R, Laviola L, Giorgino F. Human adipose tissue precursor cells: a new factor linking regulation of fat mass to obesity and type 2 diabetes? Arch Physiol Biochem (2009) 115(4):218-26. doi:10.1080/ 13813450903260864

30. Majka SM, Barak Y, Klemm DJ. Concise review: adipocyte origins: weighing the possibilities. Stem Cells (2011) 29(7):1034-40. doi:10.1002/stem.653

31. Chau YY, Bandiera R, Serrels A, Martinez-Estrada OM, Qing W, Lee M, et al. Visceral and subcutaneous fat have different origins and evidence supports a mesothelial source. Nat Cell Biol (2014) 16(4):367-75. doi:10.1038/ ncb2922

32. Min SY, Kady J, Nam M, Rojas-Rodriguez R, Berkenwald A, Kim JH, et al. Human 'brite/beige' adipocytes develop from capillary networks, and their implantation improves metabolic homeostasis in mice. Nat Med (2016) 22(3): 312-8. doi:10.1038/nm.4031

33. Misso ML, Murata Y, Boon WC, Jones ME, Britt KL, Simpson ER. Cellular and molecular characterization of the adipose phenotype of the aromatasedeficient mouse. Endocrinology (2003) 144(4):1474-80. doi:10.1210/en.2002221123

34. Van Sinderen ML, Steinberg GR, Jorgensen SB, Honeyman J, Chow JD, Herridge KA, et al. Effects of estrogens on adipokines and glucose homeostasis in female aromatase knockout mice. PLoS One (2015) 10(8):e0136143. doi:10.1371/journal.pone. 0136143

35. Witte MM, Resuehr D, Chandler AR, Mehle AK, Overton JM. Female mice and rats exhibit species-specific metabolic and behavioral responses to ovariectomy. Gen Comp Endocrinol (2010) 166(3):520-8. doi:10.1016/j.ygcen. 2010.01.006

36. Geary N, Asarian L, Korach KS, Pfaff DW, Ogawa S. Deficits in E2-dependent control of feeding, weight gain, and cholecystokinin satiation in ER-alpha null mice. Endocrinology (2001) 142(11):4751-7. doi:10.1210/endo.142. 11.8504

37. Syed FA, Fraser DG, Monroe DG, Khosla S. Distinct effects of loss of classical estrogen receptor signaling versus complete deletion of estrogen receptor alpha on bone. Bone (2011) 49(2):208-16. doi:10.1016/j.bone.2011. 03.771

38. Antonson P, Omoto Y, Humire P, Gustafsson JA. Generation of ERalphafloxed and knockout mice using the Cre/LoxP system. Biochem Biophys Res Commun (2012) 424(4):710-6. doi:10.1016/j.bbrc.2012.07.016

39. Manrique C, Lastra G, Habibi J, Mugerfeld I, Garro M, Sowers JR. Loss of estrogen receptor alpha signaling leads to insulin resistance and obesity in young and adult female mice. Cardiorenal Med (2012) 2(3):200-10. doi:10.1159/000339563

40. Vieira Potter VJ, Strissel KJ, Xie C, Chang E, Bennett G, Defuria J, et al. Adipose tissue inflammation and reduced insulin sensitivity in ovariectomized mice occurs in the absence of increased adiposity. Endocrinology (2012) 153(9):4266-77. doi:10.1210/en.2011-2006

41. Shen M, Kumar SP, Shi H. Estradiol regulates insulin signaling and inflammation in adipose tissue. Horm Mol Biol Clin Investig (2014) 17(2):99-107. doi:10.1515/hmbci-2014-0007

42. Davis KE, Neinast MD, Sun K, Skiles WM, Bills JD, Zehr JA, et al. The sexually dimorphic role of adipose and adipocyte estrogen receptors in modulating adipose tissue expansion, inflammation, and fibrosis. Mol Metab (2013) 2(3):227-42. doi:10.1016/j.molmet.2013.05.006 
43. Ribas V, Drew BG, Le JA, Soleymani T, Daraei P, Sitz D, et al. Myeloid-specific estrogen receptor alpha deficiency impairs metabolic homeostasis and accelerates atherosclerotic lesion development. Proc Natl Acad Sci U S A (2011) 108(39):16457-62. doi:10.1073/pnas.1104533108

44. Nilsson ME, Vandenput L, Tivesten A, Norlen AK, Lagerquist MK, WindahlSH, et al. Measurement of a comprehensive sex steroid profile in rodent serum by high-sensitive gas chromatography-tandem mass spectrometry. Endocrinology (2015) 156(7):2492-502. doi:10.1210/en.2014-1890

45. Naaz A, Zakroczymski M, Heine P, Taylor J, Saunders P, Lubahn D, et al. Effect of ovariectomy on adipose tissue of mice in the absence of estrogen receptor alpha (ERalpha): a potential role for estrogen receptor beta (ERbeta). Horm Metab Res (2002) 34(11-12):758-63. doi:10.1055/s-2002-38259

46. Karas RH, Schulten H, Pare G, Aronovitz MJ, Ohlsson C, Gustafsson JA, et al. Effects of estrogen on the vascular injury response in estrogen receptor alpha, beta (double) knockout mice. Circ Res (2001) 89(6):534-9. doi:10.1161/ hh1801.097239

47. D’Eon TM, Souza SC, Aronovitz M, Obin MS, Fried SK, Greenberg AS. Estrogen regulation of adiposity and fuel partitioning. Evidence of genomic and non-genomic regulation of lipogenic and oxidative pathways. J Biol Chem (2005) 280(43):35983-91. doi:10.1074/jbc.M507339200

48. Fatima LA, Campello RS, Santos RS, Freitas HS, Frank AP, Machado UF, et al. Estrogen receptor 1 (ESR1) regulates VEGFA in adipose tissue. Sci Rep (2017) 7(1):16716. doi:10.1038/s41598-017-16686-7

Conflict of Interest Statement: The authors declare that the research was conducted in the absence of any commercial or financial relationships that could be construed as a potential conflict of interest.

The reviewer KM and handling Editor declared their shared affiliation.

Copyright (C) 2018 Gavin, Sullivan, Kohrt, Majka and Klemm. This is an open-access article distributed under the terms of the Creative Commons Attribution License (CC BY). The use, distribution or reproduction in other forums is permitted, provided the original author(s) and the copyright owner are credited and that the original publication in this journal is cited, in accordance with accepted academic practice. No use, distribution or reproduction is permitted which does not comply with these terms. 